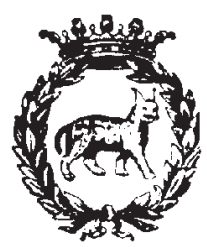

Algebraic Geometry - Points of order two on theta divisors, by VALERIA Ornella Marcucci and Gian Pietro Pirola, communicated on 9 March 2012.

Abstract. - We give a bound on the number of points of order two on the theta divisor of a principally polarized abelian variety $A$. When $A$ is the Jacobian of a curve $C$ the result can be applied in estimating the number of effective square roots of a fixed line bundle on $C$.

KEY WORDS: Abelian variety, theta divisor, torsion points.

Mathematics Subject Classification: 14K25 (primary); 14H40 (secondary).

\title{
INTRODUCTION
}

In this paper we give an upper bound on the number of 2-torsion points lying on a theta divisor of a principally polarized abelian variety. Given any principally polarized abelian variety $A$ of dimension $g$ and symmetric theta divisor $\Theta \subset A$, $\Theta$ contains at least $2^{g-1}\left(2^{g}-1\right)$ points of order two, the odd theta characteristics. Moreover, in [Mum66] and [Igu72, Chapter IV, Section 5] it is proved that $\Theta$ cannot contain all points of order two on $A$.

In this work we use the projective representation of the theta group to prove the following:

Given a principally polarized abelian variety $A$, any translated $t_{a}^{*} \Theta$ of a theta divisor $\Theta \subset A$ contains at most $2^{2 g}-2^{g}$ points of order $2\left(2^{2 g}-(g+1) 2^{g}\right.$ if $t_{a}^{*} \Theta$ is irreducible and not symmetric).

Our bound is far from being sharp. In [SM94] Salvati Manni proved that there are at least $g(2 g+1)$ points of order two outside an irreducible theta divisor. This gives a better bound for $g<7$. We conjecture that the right estimate should be $2^{2 g}-3^{g}$ as in the case of a product of elliptic curves.

When $A$ is the Jacobian of a curve $C$ the result can be applied in estimating the number of effective square roots of a fixed line bundle on $C$ (cf. Section 2).

This work has been partially supported by 1) FAR 2012 (PV) "Varietà algebriche, calcolo algebrico, grafi orientati e topologici” 2) INdAM (GNSAGA) 3) PRIN 2009 "Moduli, strutture geometriche e loro applicazioni". 


\section{MAin ReSUlT}

In this section we prove our main result.

THEOREM 1.1. Let $A$ be a principally polarized abelian variety of dimension $g$ and let $\Theta$ be a symmetric theta divisor.

1. For each $a \in A$ there are at most $2^{2 g}-2^{g}$ points of order two lying on $t_{a}^{*} \Theta$.

2. Let $a \in A$ and assume that $\Theta$ is irreducible and $t_{a}^{*} \Theta$ is not symmetric with respect to the origin. Then there are at most $2^{2 g}-(g+1) 2^{g}$ points of order two lying on $t_{a}^{*} \Theta$.

Proof. Denote by $(K,\langle\cdot, \cdot\rangle)$ the group of 2-torsion points on $A$ with the perfect pairing induced by the polarization. Let

$$
\left\{a_{1}, \ldots, a_{g}, b_{1}, \ldots, b_{g}\right\}
$$

be a basis of $K$ over the field of order two such that

$$
\left\langle a_{i}, b_{j}\right\rangle=\delta_{i j}, \quad\left\langle a_{i}, a_{j}\right\rangle=0, \quad\left\langle b_{i}, b_{j}\right\rangle=0
$$

and let

$$
H:=\left\langle a_{1}, \ldots, a_{g}\right\rangle
$$

be the subgroup of $K$ generated by the elements $a_{1}, \ldots, a_{g}$. Consider the projective morphism $\varphi: A \rightarrow \mathbb{P}^{2^{g}-1}$ associated to the divisor $2 \Theta$. By the construction of the projective representation of the theta group $K(2 \Theta)$ (see [Mum66], [Kem91, Chapter 4] and [Kem89]), we know that the elements of $\varphi(H)$ are a basis of the projective space. In the same way, the images of the elements of a coset $H_{b}$ of $H$ in $K$ generate the projective space $\mathbb{P}^{2^{g}-1}$.

Suppose by contradiction that there exists a subset $S \subset K$ such that all points of $S$ lie on $t_{a}^{*} \Theta$ and $|S|>2^{2 g}-2^{g}$. By the previous argument, since $H_{b} \subset S$ for some $b$, the points of $\varphi(S)$ generate the entire projective space $\mathbb{P}^{2^{g}-1}$. On the other hand, by the Theorem of the Square ([Mum08, Chapter II, Section 6, Corollary 4]),

$$
t_{a}^{*} \Theta+t_{-a}^{*} \Theta \equiv 2 \Theta
$$

It follows that the points of $\varphi(S)$ lie on an hyperplane of $\mathbb{P}^{2^{g}-1}$. This proves $(1)$.

Now we prove the second part. Suppose by contradiction that there exists a subset $S \subset K$ such that all points of $S$ lie on $t_{a}^{*} \Theta$ and $|S|>2^{2 g}-(g+1) 2^{g}$. We claim that

$$
\text { the points in } \varphi(S) \text { lie on a } 2^{g}-g-2 \text {-plane in } \mathbb{P}^{2^{g}-1} \text {. }
$$


Given a point $\varepsilon \in S$, it holds also $\varepsilon \in t_{-a}^{*} \Theta$. Thus $S \subset t_{a}^{*} \Theta \cap t_{-a}^{*} \Theta$. If $t_{a}^{*} \Theta$ is not symmetric and irreducible, $t_{a}^{*} \Theta \cap t_{-a}^{*} \Theta$ has codimension 2 in $A$ and we can consider the natural exact sequence

$$
0 \rightarrow \mathcal{O}_{A}(-2 \Theta) \rightarrow \mathcal{O}_{A}\left(-t_{-a}^{*} \Theta\right) \oplus \mathcal{O}_{A}\left(-t_{a}^{*} \Theta\right) \rightarrow I_{t_{a}^{*} \Theta \cap t_{-a}^{*} \Theta} \rightarrow 0
$$

by tensoring it with $\mathcal{O}_{A}(2 \Theta)$ we get

$$
0 \rightarrow \mathcal{O}_{A} \rightarrow \mathcal{O}_{A}\left(t_{a}^{*} \Theta\right) \oplus \mathcal{O}_{A}\left(t_{-a}^{*} \Theta\right) \rightarrow I_{t_{a}^{*} \Theta \cap t_{-a}^{*} \Theta} \otimes \mathcal{O}_{A}(2 \Theta) \rightarrow 0 .
$$

Passing to the corresponding sequence on the global sections, we have

$$
\begin{aligned}
0 & \rightarrow H^{0}\left(A, \mathcal{O}_{A}\right) \rightarrow H^{0}\left(A, \mathcal{O}_{A}\left(t_{a}^{*} \Theta\right)\right) \oplus H^{0}\left(A, \mathcal{O}_{A}\left(t_{-a}^{*} \Theta\right)\right) \\
& \rightarrow H^{0}\left(I_{t_{a}^{*} \Theta \cap t_{-a}^{*} \Theta} \otimes \mathcal{O}_{A}(2 \Theta)\right) \rightarrow H^{1}\left(A, \mathcal{O}_{A}\right) \rightarrow 0,
\end{aligned}
$$

since, by the Kodaira vanishing theorem (see e.g. [GH94, Chapter 1, Section 2]),

$$
H^{1}\left(A, \mathcal{O}_{A}\left(t_{a}^{*} \Theta\right)\right)=H^{1}\left(A, \mathcal{O}_{A}\left(t_{-a}^{*} \Theta\right)\right)=0 .
$$

It follows that

$$
\operatorname{dim} H^{0}\left(I_{t_{a}^{*} \Theta \cap t_{-a}^{*} \Theta} \otimes \mathcal{O}_{A}(2 \Theta)\right)=g+1 .
$$

Thus the points in $\varphi\left(t_{a}^{*} \Theta \cap t_{-a}^{*} \Theta\right)$ lie on a $2^{g}-g-2$-plane of $\mathbb{P}^{2^{g}-1}$ and the claim $(*)$ is proved.

To conclude the proof of (2) we notice that if $|S|>2^{2 g}-(g+1) 2^{g}$ then $\left|S \cap H_{b}\right|>2^{g}-(g+1)$ for some coset $H_{b}$ of $H$ (see (1)). Then it follows that $\varphi(S)$ contains at least $2^{g}-g$ independent points and we get a contradiction.

REMARK 1.2. One might expect the right bound to be $2^{2 g}-3^{g}$ and that this is realized only in the case of a product of elliptic curves.

REMARK 1.3. The argument of Theorem 1.1 can be also used to obtain a bound on the number of $n$-torsion points (with $n>2$ ) lying on a theta divisor.

\section{Applications}

In this section we apply Theorem 1.1 to the case of Jacobians. This gives a generalization of [MP, Proposition 2.5].

Proposition 2.1. Let $C$ be a curve of genus $g$ and $M$ be a line bundle of degree $d \leq g-1$. Given an integer $k \leq g-1-d$, for each $L \in \operatorname{Pic}^{2 k}(C)$ there are at least $2^{g}$ line bundles $\eta \in \operatorname{Pic}^{k}(C)$ such that $\eta^{2} \simeq L$ and $h^{0}(\eta \otimes M)=0$.

Proof. We prove the statement for $M \simeq \mathscr{O}_{C}$ and $k=g-1$. The general case follows from this by replacing $L$ with $M^{2} \otimes L \otimes \mathcal{O}_{C}(p)^{2 n}$, where $p$ is an arbitrary 
point of $C$ and $n:=g-1-k-d$. Denote by $\Theta$ the divisor of effective line bundles of degree $g-1$ in $\operatorname{Pic}^{g-1}(C)$. Given the morphism

$$
\begin{aligned}
m_{2}: \operatorname{Pic}^{g-1}(C) & \rightarrow \operatorname{Pic}^{2 g-2}(C) \\
\eta & \mapsto \eta^{2}
\end{aligned}
$$

we want to prove that $\left|m_{2}^{-1}(L) \cap \Theta\right| \leq 2^{2 g}-2^{g}$. Let $\alpha \in m_{2}^{-1}(L)$, we have

$$
m_{2}^{-1}(L)=\left\{\alpha \otimes \sigma \text { s.t. } \sigma^{2}=\mathcal{O}_{C}\right\} .
$$

If $\left|m_{2}^{-1}(L) \cap \Theta\right|>2^{2 g}-2^{g}$, then there are more than $2^{2 g}-2^{g}$ points of order two lying on a translated of a symmetric theta divisor of $J(C)$ and, by (1) of Theorem 1.1, we get a contradiction.

REMARK 2.2. If we apply Proposition 2.1 to $M=\mathcal{O}_{C}, L=\omega_{C}$, we get that on a curve of genus $g$ there are at most $2^{2 g}-2^{g}$ effective theta characteristics. We notice that when $g=2$ they are the 6 line bundles of type $\mathcal{O}_{C}(p)$ where $p$ is a Weierstrass point. When $g=3$ and $C$ is not hyperelliptic, they correspond to the 28 bi-tangent lines to the canonical curve.

COROLlary 2.3. Let $C$ be a curve of genus $g$ and $M_{1}, \ldots M_{N}$ be a finite number of line bundles of degree $d \leq g-1$. Given an integer $k \leq g-1-d$, if $\eta$ is $a$ generic line bundle of degree $k$ such that $h^{0}\left(\eta^{2}\right)>0$, then

$$
h^{0}\left(\eta \otimes M_{i}\right)=0 \quad \forall i=1, \ldots, N .
$$

ProOF. Let

$$
\Lambda:=\left\{\eta \in \operatorname{Pic}^{k}(C): h^{0}\left(\eta^{2}\right)>0\right\},
$$

and, for each $i=1, \ldots N$, consider its closed subset

$$
\Lambda_{i}:=\left\{\eta \in \Lambda: h^{0}\left(M_{i} \otimes \eta\right)>0\right\} .
$$

We remark that $\Lambda$ is a connected $2^{2 g}$-étale covering of the image of the $2 k$-th symmetric product of $C$ in $\operatorname{Pic}^{2 k}(C)$. By Proposition 2.1, for each effective $L \in \operatorname{Pic}^{2 k}(C)$ there exists $\eta \in \Lambda \backslash \Lambda_{i}$ such that $\eta^{2} \simeq L$. It follows that $\Lambda_{i}$ is a proper subset of $\Lambda$. Since $\Lambda$ is irreducible, also the set

$$
\bigcup_{i=1}^{N} \Lambda_{i}=\left\{\eta \in \operatorname{Pic}^{k}(C): h^{0}\left(M_{i} \otimes \eta\right)>0 \text { for some } i\right\}
$$

is a proper closed subset of $\Lambda$.

Acknowledgements. We would like to thank Riccardo Salvati Manni for his valuable comments and remarks. 


\section{REFERENCES}

[GH94] P. Griffiths - J. Harris, Principles of algebraic geometry, Wiley Classics Library. John Wiley \& Sons Inc., New York, 1994. Reprint of the 1978 original.

[Igu72] J. Igusa, Theta functions, Springer-Verlag, New York, 1972. Die Grundlehren der mathematischen Wissenschaften, Band 194.

[Kem89] G. R. KempF, The addition theorem for abstract theta functions, in Algebraic geometry and complex analysis (Pátzcuaro, 1987), volume 1414 of Lecture Notes in Math., pages 1-14. Springer, Berlin, 1989.

[Kem91] G. R. Kempf, Complex abelian varieties and theta functions, Universitext, Springer-Verlag, Berlin, 1991.

[MP] V. Marcucci - G. P. Pirola, Generic Torelli theorem for Prym varieties of ramified coverings, Compositio Math., to appear, arXiv:1010.4483v3.

[Mum66] D. Mumford, On the equations defining abelian varieties, I, Invent. Math. 1:287354, 1966.

[Mum08] D. Mumford, Abelian varieties, volume 5 of Tata Institute of Fundamental Research Studies in Mathematics. Published for the Tata Institute of Fundamental Research, Bombay, 2008.

[SM94] R. Salvati Manni, Modular varieties with level 2 theta structure, Amer. J. Math. 116(6):1489-1511, 1994.

Received 10 February 2012,

and in revised form 14 February 2012.

Valeria Ornella Marcucci Dipartimento di Matematica "F. Casorati"

Università di Pavia via Ferrata 1, 27100 Pavia Italy valeria.marcucci@unipv.it

Gian Pietro Pirola Dipartimento di Matematica "F. Casorati" Università di Pavia via Ferrata 1, 27100 Pavia

Italy gianpietro.pirola@unipv.it 
